[Special Issue on Research and Social Justice]

\title{
Toward Best Practices in Quantitative, Qualitative, and Mixed- Method Research: A Social Justice Perspective
}

\author{
Ruth Fassinger \\ John F. Kennedy University \\ Susan L. Morrow \\ University of Utah
}

\begin{abstract}
Various research methods can be appropriate for social justice aims. Quantitative, qualitative, and mixed-method approaches offer different kinds of strengths in advancing a social justice agenda. This article recaptures and expands upon the ideas presented by the authors of this special issue, recommending best practices in research for social justice in the following areas: (a) cultural competence and the role of the researcher(s); (b) formulating the focus of the research; (c) selection of the underlying paradigm and research method/design; (d) the research team: formation, process, and issues of power; (e) power and relationship with research participants; and ( $f$ ) data gathering, analysis, and reporting.
\end{abstract}

Keywords: Social justice, qualitative research, quantitative research, mixed-method research

\section{Introduction}

Research in counseling and psychology has focused increasingly on diversity and multiculturalism. More recently, the social justice perspective that grounded the field at the turn of the 20th century has been revived and also is receiving increased attention (e.g., Toporek, Gerstein, Fouad, Roysircar, \& Israel, 2006). Concurrently, feminist and multicultural scholars have criticized much of the existing literature for its lack of attention to the needs and issues of populations currently marginalized in society (and in psychology) based on such status factors as race, ethnicity, gender, sexual orientation, disability, immigration status, and religion 
(e.g., Sue, 1999). From these critiques has emerged a greater scholarly focus on investigating the effects of systematic forms of inequity and oppression, and a concurrent desire to empower marginalized groups through socially just research practices. This special issue is aimed at articulating ways in which quantitative, qualitative, and mixed-method research approaches can serve social justice goals.

In this article, we reflect on the three perspectives presented in the special issue, using them as a basis for suggesting best practices for social justice research in counseling and psychology. Foundational to our discussion is an assumption (virtually axiomatic in feminist and multicultural methodological discourse; see Harding, 1986; Unger, 1983 for now-classic statements) that, while research methods have implicit philosophies of science embedded in them that reflect values regarding knowledge and human behavior, users of these methods also infuse their investigations with values that render those investigations non-neutral. That is, scientific exploration is never value-free, regardless of methodological approach, but is culturally and temporally situated, enacted by human beings who bring their own unique lenses to each research endeavor. The attitudes, biases, and worldviews of researchers permeate their work from beginning to end, and, just as any method can be used to justify existing social inequities, any method "can be liberating if used by multi-culturally competent researchers and scholaractivists committed to social justice" (Cokley \& Awad, 2013, p. 26). That is, research can be used either to perpetuate or to disrupt the social status quo, to oppress or to empower marginalized groups, to provide an experience that blames people for their victimization or seeks to liberate them and transform their lives. It is not the method alone that determines the outcome, but rather the intention behind and the use of that method to support social justice aims.

We note here that our own lenses through which we view and comment upon these issues have been informed by our feminist, multicultural perspectives, as well as by our experiences as scholars, clinicians, educators, and social activists who have embraced a wide range of research approaches during our careers. We view a social justice perspective, by its very focus on marginalized cultural groups and its explicit goal of social change, as a framework that can transform any research method into a socially just endeavor in the hands of a multi-culturally and methodologically competent researcher. We see the possibilities for social justice research on a continuum ranging from investigations that build knowledge and raise awareness to research that engages both researchers and participants in social action projects whose goal is changing oppressive systems and structures. On this continuum, the impact of a research project aimed at social change may result in any of the following: (a) the publication and dissemination of a quantitative, qualitative, or mixed-method study becomes a catalyst for social change through its knowledge generation and its consciousness-raising impact on audiences; (b) the respectful and non-deceptive treatment of research participants contributes to their gaining voice and becoming empowered through the research process; (c) participants' agendas become the central focus of the research, with the investigator serving as a consultant; or (d) the research project itself is a social change intervention, in which researchers and participants collaborate.

The authors of this special issue have presented many ways in which quantitative, qualitative, and mixed-method research can contribute to a social justice agenda. We have drawn on their ideas and insights in identifying best practices in research for social justice in counseling and psychology. We have organized our discussion according to practices in the following areas: (a) 
cultural competence and the role of the researcher(s); (b) formulating the focus of the research; (c) selection of the underlying paradigm and research method/design; (d) the research team: formation, process, and issues of power; (e) power and relationship with research participants; and (f) data gathering, analysis, and reporting.

\section{Cultural Competence and Role of the Researcher(s)}

All of the authors of this special issue in some way addressed the importance of multicultural skills, cultural competence, and respect for the cultural values of participants by researchers. We note that cultural competence, manifested in the ability to navigate a culture different from one's own, is a foundational necessity for all researchers, whether they are members of dominant or marginalized groups themselves. Effective multicultural counseling skills can help researchers build rapport, encourage self-reflection, and promote respectful interaction with participants. Ponterotto and Grieger (2008) identified competency areas expected of all multicultural counseling researchers, including researcher self-awareness as a cultural being; knowledge of past psychological and health research with minority populations; community consultation and responsibility; ethical research practice; and knowledge of philosophy of science and research paradigms, with bi-methodological competence in quantitative and qualitative methods (cited in Ponterotto, Mathew, and Raughley, 2013, p. 54).

Also emphasized by the authors of this special issue were the importance of understanding such details as participants' cultural histories; relevant laws and policies; values, norms, customs, and traditions of participants and their communities; and the sociopolitical climate as viewed from participants' perspectives. The researcher should possess culturally competent communication skills (including language fluency) and demonstrate respect for participants and their cultures; respect is manifested by adopting a non-pathological stance in regard to cultural manifestations and actively working to dismantle cultural stereotypes.

The authors of this special issue all emphasize the importance of cultural awareness and selfreflection on the part of the researcher(s). In socially just research, the personal experiences and perspectives of the researcher(s) are articulated, and the research processes are transparent. That is, cultural self-awareness is a cornerstone of cultural competence, and what expands cultural competence into socially-just research is the clear and public articulation of cultural self-awareness as it potentially affects the investigative process. Qualitative research approaches specifically call for researchers to make their own statuses and standpoints explicit, and to examine and expose their assumptions and biases. We would argue that this practice should be embedded in all research, and described explicitly in resulting publications. Particularly in research for social justice, in which the populations typically being studied are outside of the mainstream discourse, researchers must recognize themselves as culturallysituated beings and be acutely aware of the attitudes and biases they harbor about their own cultural group as well as other groups. It is important that they publicly declaim their awareness of their own cultural worldviews, and attempt to situate them in relation to the worldviews of the participants with whom they interact.

In addition, it is essential for researchers to examine their motivations for conducting the planned research, and this is where the "insider/outsider" status of the researcher can come into play. If the researcher comes to the population under study as a cultural outsider (and, 
due to advanced education, possessing certain privilege and status regardless of personal circumstances), she or he must consider possible perceptions of cultural mistrust on the part of research participants. If there are large gaps in status between researcher and participants (e.g., a senior academic white female investigating achievement behaviors of poor, African American female teens), attitudes stemming from researcher privilege may take the form of being patronizing, overprotective, or wanting to "save" those with less advantage. Outsider researchers must grapple with the fundamental question of whether there are certain kinds of research that should not be conducted by those in particular positions of privilege and power because of the potential for perpetuating oppression and micro-aggressions. Situations in which such research may prove impossible include conditions where cultural mistrust is high, stereotypes abound, there has been a history of exploitation of a people by researchers, sovereignty is an issue, or highly sensitive and culturally-contextualized concerns (e.g., spirituality or sexuality) are central to the investigation. For example, a senior white male academic may expect to encounter so many barriers in investigating the sexual abuse of Latina females that the research is deemed impossible to implement, unless a research collaborator or team is used (see below).

On the other hand, insider researchers also face challenges. Like outsiders, cultural insiders must examine their own life experiences and worldviews to anticipate ways in which their experiences may affect the research. Given that communities are made up of highly diverse individuals, it is likely that a researcher's own experiences will differ markedly from those of many other members of the community, even when the researcher appears to be a credible member of the community. Although it is tempting to assume some degree of common experience, this assumption may be misleading and can seriously distort or compromise the research; for example, a lesbian researcher who wishes to study the coming out process in lesbians and assumes her own experience to be typical will likely miss or misinterpret conflicting data. In addition, cultural insiders are not always recognized as "inside" the community by others, and their own self-definition may not reflect the perceptions of their potential research participants; again, returning to the lesbian studying other lesbians, if she is known in her community to be extremely closeted or she is not even known in the community as a lesbian, she is likely to encounter resistance and disrespect from some potential participants.

One way to address both insider and outsider concerns in socially just research is to use a diverse and collaborative team of researchers who represent those both within and outside of the community of focus. This can be particularly helpful when a researcher lacks a status characteristic (e.g., Latino/a), identification (e.g., LGBT), or skill (e.g., language fluency) that would make a research endeavor challenging or impossible individually. A research team also can and should engage in a continuous self-reflective process that brings strong feelings and deeply ingrained attitudes to light so that they can be addressed appropriately during the conduct of the research. Particularly in analytic processes such as coding narrative data or interpreting puzzling statistical patterns, the benefits of multiple perspectives from diverselysituated collaborators can make all the difference in whether an important question, idea, or speculation receives the attention it deserves in coming to understand a phenomenon of interest. For example, in a study of highly achieving Latinas (Gomez, Fassinger, Prosser, Cooke, Mejia, \& Luna, 2001), one of us (a white female) utilized a diverse research team including several women of color. Their recognition of the strong subcultures among Latinas proved invaluable in highlighting in-population variability during sampling decisions as well as data analysis and interpretation. 
In summary, recommended best practices related to researcher role and competency are:

1. Researchers undertaking research with social justice aims should understand that such research demands multicultural competency as a necessary (but not sufficient) condition for entering into an investigative process-that social justice research is cultural research.

2. Researchers should understand their own status and position as culturally-situated beings-particularly regarding power and privilege-in relation to the community under study, and be prepared to honor the strengths and support the needs of that community as they become manifested during the course of the research.

\section{Formulating the Focus of the Research}

Researchers may follow traditional processes to define the focus of the research, including examining the existing literature to identify possible topics and research questions that may contribute to social justice goals. However, as the authors in this special issue point out, it also is important to bring attention to people and issues that have been underrepresented in the scholarly literature, and this may require a different kind of approach-one typically used in certain qualitative methods-in which the researcher enters a participant community with an open and inquiring stance, allowing specific research directions and questions to emerge from the needs, concerns, and voices of participants themselves. In actual practice, of course, gaining clarity about research questions often occurs through an iterative process of data gathering and continually returning to the literature. This is true of quantitative studies as well as qualitative and mixed-method investigations, regardless of how simplistically the research process is rendered in published accounts.

Social justice researchers often find themselves in a conundrum when weighing sources of knowledge where there are conflicting perspectives, such as the extant academic literature versus the cultural wisdom of the people being studied. Cultural wisdom or knowledge is extraordinarily difficult to articulate, as cultures or communities are composed of diverse individuals who may present differing perspectives on phenomena under study, and a sense of collective agreement may be challenging to discover-an exercise that forms the basis of several qualitative methods, such as grounded theory. Moreover, cultural knowledge-as gleaned from anecdotal, observational, experiential, and narrative data-often lacks legitimacy in the eyes of the academy, and too many "personal communication" citations in a manuscript are likely to raise suspicion in reviewers. Thus, one of the first challenges facing social justice researchers is to find ways to frame scientific study in a way that legitimizes cultural knowledge. Typically, it is those with some degree of power and privilege who are permitted to engage in this kind of legitimization, and this offers another justification for collaborative research teams in which there are varying degrees of seniority and academic "clout."

The authors of this special issue, as well as other qualitative and mixed methods researchers and methodologists, have identified additional considerations for the focus of social justice research. Lyons, Johnson, Bike, Flores, Ojeda, and Rosales (2013) noted that qualitative research, in particular, enables researchers to gain a perspective that uncovers the meanings of individual experiences from within a cultural context. This cultural contextualization is one of 
the cornerstones of social justice research. Cokley and Awad (2013) assert that social justice research serves advocacy and policy goals and that quantitative data may be especially persuasive in the policy arena. These authors also note the value in socially just research of introducing "empirical constructs which are rooted in the values and realities of a marginalized and/or oppressed population" (p. 30) that is, variables tapping specific cultural or community characteristics or issues (such as "familismo" in Latino populations or "internalized homophobia" in LGBT communities), so that research ultimately becomes more inclusive of marginalized groups.

Morrow (2005) suggested that topics selected for investigation should have obvious (not contrived) practical utility, and all of the authors writing in this special issue agree that the main foundational assumption of social justice research is that its ultimate goal is social change-that the primary benefit of such research should accrue to those being studied, and that the needs of and potential benefits to the community should constitute the major priority of the researcher(s) undertaking social justice studies. In addition, Cokley and Awad (2013) point out the importance of considering possible negative consequences of the research on the population under study, particularly in light of the oppressive uses of psychological research historically. One example of negative consequences would be methodological decisions that interfere with the collectivist harmony of the community. Indeed, Lyons et al. (2013) assert that an "individualism-focused" research approach may be "markedly inconsistent with the worldview of certain collectivist culture communities", where even the practice of conducting personal, private interviews would be viewed as-or actually be-disruptive of the social fabric of the community. Other examples of possible negative research impact include results suggesting interventions that are prohibitively costly or discovering that a highly-prized community activity is ineffective or even harmful to some or many members of the community. Recent reports suggesting the ineffectiveness of abstinence-only sex education programs provide examples of findings widely decried in religious communities that value and promote such programs among their youth.

In summary, best practices emanating from framing social justice research are:

3. Researchers should understand the importance of framing social justice research with the needs and desires of and benefits to the community under study as the primary aim.

4. Researchers should understand the importance of framing research in ways that legitimize cultural knowledge and social change goals.

\section{Selection of Underlying Paradigm and Research Method / Design}

As we noted above, it is our belief that any research method can be used for socially just (or unjust) purposes, and we concur with the preference of Ponterotto et al. (2013) for "methodological situationalism", which values both quantitative and qualitative methods and urges that the choice of method be dictated by the particular research questions at hand and the state of knowledge regarding that area of study. As these authors point out, strengths emanate from both approaches regarding cultural research with social justice aims. Qualitative approaches can help to: enhance relationship and dialogue between researchers and 
participants in their communities; minimize the imposition of researcher assumptions on diverse others; empower participants by helping them to voice their stories and by honoring their strengths, needs, and values; stimulate collaborative social change efforts by researchers and participants; catalyze theory development; and frame communication and dissemination of research outcomes in ways that are immediately useful to communities.

Quantitative approaches, on the other hand, can help to: provide large, representative samples of cultural communities; reliably assert cause-and-effect relationships among constructs as well as confirm or disconfirm theoretical hypotheses; and summarize numerical data in ways that are clear and persuasive to leaders and policy-makers. Mixed-method approaches, which have the potential to combine the best of both quantitative and qualitative methods, "can compensate for uni-paradigmatic limitations" (Ponterotto et al., 2013, p. 47) and offer researchers maximum flexibility. Although the paradigmatic underpinnings of quantitative and qualitative approaches may differ substantively enough that they appear to be incompatible, Ponterotto et al. (2013) argue that adequate researcher knowledge of both approaches can help to ensure effective conceptualization and implementation of mixed-method designs in ways that preserve the strengths of each in any given research endeavor. We would further assert that social justice research, by its very nature as a contextually-driven, multiculturally-oriented effort toward social transformation, can create a unifying thread in mixed-method approaches; it helps to ensure that foundational assumptions regarding research goals, ways of interacting with the community, care in analyzing and interpreting data, and communicating results publicly align with both the quantitative and qualitative elements of a mixed-method study.

We would further argue that the basic goals of social justice research-to examine and understand systematic social inequities that privilege or marginalize particular groups of people, and to work toward social change that results in the re-distribution of power and resources-are particularly compatible with a critical/ideological paradigm that is explicitly value-laden and "serves to disrupt and challenge the status quo" (Ponterotto, 2005, p 129). Issues of power and oppression are central to this paradigm, and research that follows from it not only supports a relational approach to the conduct of research but also provides the opportunity to engage what Guba and Lincoln (1989) termed authenticity criteria. These criteria-fairness, ontological authenticity, educative authenticity, and catalytic authenticity-have particular relevance for social justice research. Authenticity criteria include soliciting and honoring different perspectives; facilitating the maturation and expansion of participants' perspectives during the research process; facilitating the understanding by participants of one another's constructions; and utilizing the research endeavor as stimulation to action.

Many forms of research can emanate from a critical-ideological paradigm, but probably the best-known approach is Participatory Action Research (PAR), which has as its explicit and primary goal empowering a community toward social change. The central question asked by PAR researchers is: "How are systems of power and privilege manifested in the lived experiences of this person or group of people, and how can knowledge be gained and used to raise consciousness, emancipate, and empower this person and group?" (Betz \& Fassinger, 2011 , p. x). Because PAR emerges completely from the needs of a community and has social change as a primary focus, it is an ideal approach to accomplish many social justice goals, including consciousness-raising, community action, change in the lives of participants, empowerment and liberation (Kidd \& Kral, 2005). PAR requires intensive and prolonged involvement by the researcher(s), and its success is judged by the extent and kinds of changes 
in the lives of participants (including institutional and structural elements in which their lives are embedded), an extraordinarily difficult challenge given deeply entrenched attitudinal and structural barriers to community participation and social change (Betz \& Fassinger, 2011). Although PAR is often categorized as a qualitative approach because its unabashed political stance anchors it at the subjective end of the epistemological/axiological continuum, in practice it actually is a mixed-method approach, as researchers typically utilize any forms of data and analyses that will contribute to social change goals. Overall, the PAR approach has much to offer, even if it can be implemented only partially within the constraints experienced by most researchers. We recommend that all researchers who are serious about social justice work acquire familiarity with this promising method-its radical stance will stretch researchers in imagining the possibilities of their research, and its varied activities will stimulate creativity in research design and implementation.

In summary, best practices related to selecting a research approach are:

5. Researchers should become well-versed in quantitative, qualitative, and mixedmethod approaches so that they can make reasoned choices about the most appropriate method(s) to be used in a given study.

6. Researchers are especially encouraged to obtain familiarity with Participatory Action Research for its promise in social justice research.

\section{The Research Team: Formation, Process, and Issues of Power}

Several of the authors of this special issue addressed critical considerations in the formation and use of a research team, increasingly a feature of published reports of psychological research. In addition to representing both insider/outsider statuses (mentioned above), a research team that is constituted to maximize diversity can balance strengths and limitations among teammates, provide growth for its members, challenge stereotypical thinking as well as biases and assumptions, and promote critical thinking. Interdisciplinary teams can broaden the scope of understanding of the phenomenon being studied. Teams that include expertise across a variety of research methods can help to ensure that various elements of quantitative, qualitative, and/or mixed-method approaches are incorporated appropriately and effectively. Additional inclusionary considerations suggested by the authors of this special issue address, for example, representation of professionals who are members of marginalized groups; engagement of both junior and senior researchers to provide mentoring and to increase the credibility of the research endeavor; and inclusion of community members and/or leaders as research team members who can provide incalculable benefits to the rigor and relevance of the project while also empowering research participants.

An important aspect of working in research teams is addressing issues of power and status on the team. It is important not only to engage a diverse team with a wide range of representation based on the needs of the study, but also to examine and monitor what power these team members hold to determine who gets added to the team, what analytic decisions get made, how funding is distributed, and how authorship is determined, to name but a few of the many issues of power and privilege that emerge in any collaborative effort. In addition, a data analysis and interpretation process that is ideally consensual demands that all team members 
are able to function as equals in the research environment. Lyons et al. (2013) note that it is critically important to understand how researcher inputs may influence data analysis and interpretation in a study. A diverse and democratic research team, in which all members have equal voice, can help to ensure that researcher inputs are inclusive of a broad range of perspectives, are creative and imaginative in producing out-of-the-box thinking, and that implicit biases and attitudinal assumptions have been thoroughly exposed, challenged, and addressed in the research process. Typically, such benefits will accrue through ongoing reflection, discussion, and debate among team members, so explicit attention to relative power on the research team and active efforts to equalize power and maintain balance are essential. While, ultimately, it is the responsibility of the senior researcher(s) to ensure the safety and open participation of all team members, we advise that this be accomplished collaboratively, by sharing power and modeling collegiality, respect, openness, and equality.

In summary, best practices related to the use of research teams are:

7. Research teams should be selected to maximize the appropriateness for and sensitive implementation of the research in diverse communities, which demands an inclusionary approach.

8. Researchers should understand the importance of equalizing and continually monitoring power differentials in the team so that open exchange among members is enhanced.

\section{Power and Relationship with Research Participants}

Entry into the worlds and lives of communities for the purpose of conducting research raises both challenges and possibilities, and all the authors of this special issue have addressed issues regarding the relationship between researchers and participants in socially just research. Challenges accrue because of the potential for exploitation and the greater privilege and power usually held by researchers. An important question is whether research can ever be completely non-exploitative. If so, how do researchers make it that way? If not, how do researchers minimize exploitation? Can research have aspects of exploitation and still be empowering? As Cokley and Awad (2013) noted, many research participants historically and currently have experienced exploitation and alienation at the hands of researchers.

Optimistically, we believe that research that contributes to participant and community wellbeing and empowerment has great potential to be non-exploitative. However, part of the selfreflective process for researchers must include examining the benefits they hope to gain, personally, from conducting the research. Financial gain, status, publication, and its related academic benefits such as tenure and promotion all have the potential for leading to exploitation or placing the researcher's own goals ahead of empowering research participants. Social justice researchers can be transparent about the benefits to themselves that may accrue from the research activity. They can also inquire about participant needs and possible contributions researchers might make in return. Differences in privilege and power may certainly arise when researchers are dominant culture members (by virtue of being White, middle or upper class, and highly educated, for example). However, even when researchers and participants come from similar or parallel sociopolitical locations or identity statuses, researchers 
typically hold power over research participants simply by being researchers. Again, transparency of the researcher's status, motives, goals, and processes can serve to reduce the negative impact of these power differences.

Educating potential research participants about research goals and processes can help to equalize power between researcher(s) and participants. Unfortunately, many aspects of the research process serve to keep the research endeavor shrouded in mystery. The informed consent document, for example, may use language required by an institutional review board that is inaccessible or distancing to participants who are not acquainted with academic institutional norms. Thus, giving participants information about the purposes of institutional review boards; presenting informed consent documents in alternate, accessible formats; and taking extra care to be sure participants understand their rights in the research can demystify the research process and reduce the power differential between researcher and participant. Participant empowerment also can be promoted by providing detailed information about who will be conducting the research; what data will be collected, why, and how; what questions will be asked or topics covered; what methods will be used to analyze, or make sense of, the data and how those methods can help the community; what the researcher hopes to learn in the study; and what the final outputs will be. Socially-just disclosure would include not only the typical risk/benefit statements, but researchers also would present an expanded acknowledgement of the ways that the investigative process might be difficult for, or negatively perceived by, participants, as well as realistic warnings about the manner in which outputs might be misused by others.

Lyons et al. (2013) particularly emphasized the importance of viewing social justice research as a relational activity and respectfully making contact and connecting with participants. The use of "gatekeepers" or "stakeholders" (p. 16), community members, or leaders who can vouch for and facilitate entry of outsider researchers can be particularly helpful. These authors suggested building relationships with key (e.g., religious) leaders who already have the trust of the community in the early stages of planning a study and using those connections to learn about and gain access to the community. Already being a genuinely engaged and active ally in a community prior to (and following) the study also may reduce the possibility of exploitation, may build trust, and may produce higher quality research through the acquisition of tacit knowledge of the context and people the researcher wishes to study.

At the core of respectful social justice research is the value of interpersonal relationships and serving the needs of the community in which the research is being conducted, that is, ensuring the opportunity for "meaningful participation" by the members of the community under investigation These authors explain: "Socially just researchers, therefore, would approach the conceptualization of the project, including research question development and other design activities, as an opportunity to begin building a collaborative relationship with the community being investigated" (p. 14). Lyons et al. (2013) further asserted that research that is dialogic and dialectic, that leads to growth and transformation, and that is empowering and serves as a mechanism for social justice. That is, the mutual meaning-making process that should characterize all social justice research can contribute to the empowerment of research participants. Participants are viewed and treated as research consultants and co-researchers in order to communicate respect and empower them. Ultimately, the researcher may leave the community in which she or he has conducted research. Leave-taking and giving back to the community are closely connected and communicate to participants that they are honored and 
valued beyond their contributions to the research. In some cases, researchers have ongoing commitments to their communities such that leave-taking is not an issue; however, in these cases, it is still important that the research itself have an ending and that participants experience closure with the research process. A research process that does not have clear boundaries or a definitive ending may confuse participants about the nature of their relationships with researchers or may leave them wondering why they participated in the research at all. Researchers who will be leaving the setting or community will want to give careful consideration to their goodbyes. Social justice research that is relationally oriented requires more of researchers than simply providing a summary of the results. Depending upon the particular cultural traditions of participants, gift-giving, celebrations, or other social markers may be appropriate ways to end the research relationship.

In addition to the research process itself serving the community by empowering its participant members, social justice researchers are deliberate about taking their findings back to communities, presenting the research in a form that will be useful to participants and communities, and making the results available to all community members-not just those who actively participated in the research. Community interventions, psychoeducation, ongoing focus groups for social change, workshops, ongoing consultation, and pamphlets are just a few ways to give back to the community. If the research has been formulated with a clear social action project (such as a PAR study) leading to specific change, the researcher also will evaluate the impact of the research effort on bringing about real community transformation. We also would add the caveat, rarely acknowledged in discussions of social transformation as catalyzed by research, that desired change may be enacted in many forms by empowered participants-and not only are these changes out of the direct control of the researcher(s), but they even may be abhorrent to the researcher in some way, an ethical issue that calls for attention as liberationist research approaches become more widely used and accepted.

In summary, best practices related to relationships with research participants are:

9. Researchers should understand that social justice aims demand a relational approach to engaging with participants, regardless of particular method. Participants are viewed as co-researchers or collaborators, with the detailed attention to communication and education that this stance entails.

10. Researchers should consider engaging with communities of interest well before the commencement of a study (perhaps with the assistance of community leaders as appropriate), and also should plan explicitly for giving back to that community in the dissemination of study outcomes.

\section{Data Gathering, Analysis, and Reporting}

From recruitment through dissemination, social justice researchers have myriad opportunities to communicate their respect for participants as well as understanding of issues of concern to participants' communities. This basic foundation of the social justice research enterprise has been addressed by all of the authors of this special issue in various ways. Respect for participants and their communities may take many forms. For example, in quantitative approaches to data collection, questionnaires may be made available in various formats (on- 
line, paper-and-pencil, translated into participants' natural languages, and modified for accessibility to people with disabilities). Respect in low-income communities may require recognition that many individuals work long hours, have little flexibility in their work schedules, and may have childcare issues as well. Thus, incentives or compensation and participation logistics need to be appropriately scaled to their needs. Researchers should consider issues such as living arrangements, childcare, transportation, time, and location when arranging to meet with or gather together participants. Choosing a site that is comfortable and accessible to participants, located in their communities, can facilitate recruitment, demonstrate respect, and build trust. Incentives to community partners or agencies (e.g., providing supplies or free workshops) may facilitate recruitment as well as sending a message to the community that the researcher takes its needs seriously.

There may be particular issues to be aware of with populations who are at heightened risk for discrimination, persecution, and oppression (e.g., immigrants, LGBT individuals). The researcher needs to understand the risks to participants if their confidentiality is jeopardized. Certificates of Confidentiality from federal agencies can help to allay fears, and researchers also may bring (prior to public dissemination) the actual text of their results summaries back to participants to determine whether there are any confidentiality concerns.

Specific activities used in data gathering also need to be appropriate to the cultural characteristics of the participants. Focus groups, participant observation, in-person interviews, genograms, cultural artifacts, questionnaires, and archival documents all present possibilities for data collection that can fit social justice goals. In particular, focus groups and group interviews may be especially useful in empowering participants, engaging members of a community in collective meaning making, building community support, maintaining congruence with communal values held in many collectivist cultures, neutralizing the power of the researcher, and normalizing participants' experiences. It also is possible for researchers to give participants choices about various aspects of the data collection process and to engage them in problemsolving around aspects of data gathering that may be difficult. Cokley and Awad (2013), for example, point out that snowball sampling may be the most productive approach to data gathering in many marginalized populations, and having community members assist in identifying key networks can greatly facilitate the data collection process.

If interviews are used (whether individually or in groups), the interview protocol itself should be scrutinized for the validity of cultural concepts, relevance to participants, capacity to provide rich description, and appropriateness to participants' educational and linguistic levels. Questions should be sequenced and flexible, and the researcher should practice, pilot, and seek feedback from cultural experts and insiders to maximize its effectiveness. Where the natural language of the researcher differs from that of the participant, interpreters should be chosen carefully, with not only linguistic, but also cultural competence as core considerations. Similar recommendations apply to personal assistants who may accompany people with disabilities. The researcher should be aware that, in some communities, even very qualified interpreters or assistants may be suspect if the interpreter/assistant is a community member and viewed as a possible threat to confidentiality or as someone who might pass judgment.

During and after data collection, analytic and interpretive processes begin. We reiterate here the importance of a collaborative team of researchers in facilitating comprehensive and trustworthy data analysis. Follow-ups or participant checks should be relevant and meaningful 
to participants (Lyons et al., 2013). For example, asking participants for feedback about the researcher's tentative interpretations or conclusions and requesting their own perspectives on the data can contribute to the quality and trustworthiness of the analysis and can bring the participants into an empowered position as they take ownership of the results. Social justice researchers have the opportunity-and, we would argue, the responsibility-to fully engage participants as co-researchers by explaining analytic procedures and conventions to them and supporting their own analytic and interpretive capabilities (Morrow \& Smith, 1995). Engaging participants as collaborators demystifies the research process and provides communities with skilled members who have the tools to continue working toward social change long after a particular study is completed.

Summarizing and disseminating the results or outcomes of social justice research provides a final opportunity for researchers to demonstrate their respect for participants and their responsiveness to community needs. Lyons et al. (2013), for example, call for involving the community in decisions about the communication and application of results. Cokley and Awad (2013) further assert that dissemination of results should actively counter commonly-held negative stereotypes about the community under study, thereby minimizing the possibility that research results can be misused in harmful ways. Ponterotto et al. (2013) advise inclusion of participant's voices as the most authentic, empowering, and powerful means of supporting analytic conclusions, as well as creating empathy in audiences that may lead to diminishment of stereotypes, thus contributing to social change. In addition, researchers should think creatively about meaningful forms of presentation of research outcomes beyond the traditional journal article. Presentations, workshops, pamphlets, dramatized performances (e.g., theatre of the oppressed), and other alternative approaches can engage whole communities in understanding what their participation has meant and what the research has revealed about their lives. In addition, such activities can serve as a bridge to further social change actions within the community, thus fulfilling the primary goal of social justice research.

In summary, best practices regarding data gathering, analysis, and reporting are:

11. Researchers should ensure that they are continually demonstrating their respect for the communities they are studying through their accommodating practices.

12. Researchers should involve their participants in data collection, analysis, and reporting, which gives them tools and skills they can use in future social change efforts.

13. Cultural experts or insiders can be used to ensure culturally- appropriate practices.

14. Researchers should use dissemination activities as a means of advocating for marginalized communities, both within the community and with outside audiences.

\section{Conclusion}

This synopsis of best practices in social justice research, as framed by the authors of this special issue, is not exhaustive by any means. However, our hope is that these ideas will inspire continued dialogue among social justice researchers in counseling and psychology, and that 
they ultimately will lead to emancipatory and empowering research practices that catalyze social change needed for a more socially just world.

\section{Contact information:}

Dr. Ruth E. Fassinger, Dean of the College of Professional Studies, John F. Kennedy University Email: rfassing@umd.edu

Susan L. Morrow, Ph.D., Professor \& Director of Clinical Training, Counseling Psychology Program, University of Utah

\section{References}

Betz, N. E., \& Fassinger, R.E. (2011). Research methodologies. In E.M. Altmaier \& J.C. Hansen (Eds.), The Oxford handbook of counseling psychology (p. 237-269). New York: Oxford University Press.

Cokley, K., \& Awad, G.H. (2013). In defense of quantitative methods: Using the "Master's Tools" to promote social justice. Journal for Social Action in Counseling and Psychology, $5(2), 26-41$.

Gomez, M. J., Fassinger, R. E., Prosser, J., Cooke, K., Mejia, B., \& Luna, J. (2001). Voces abriendo caminos (voices forging paths): A qualitative study of the career development of notable Latinas. Journal of Counseling Psychology, 48, 286-300.

Guba, E. G., \& Lincoln, Y. S. (1989). Fourth generation evaluation. Newbury Park, CA: Sage.

Harding, S. (1986). The science question in feminism. Ithaca, NY: Cornell University Press.

Kidd, S. A., \& Kral, M. J. (2005). Practicing participatory action research. Journal of Counseling Psychology, 52, 187-195.

Lyons, H. Z., Johnson, A., Bike, D. H., Flores, L. Y., Ojeda, L., \& Rosales, R. (2013). Qualitative research as social justice practice with culturally diverse populations. Journal for Social Action in Counseling and Psychology, 5(2), 10-25.

Morrow, S. L. (2005). Quality and trustworthiness in qualitative research in counseling psychology. Journal of Counseling Psychology, 52, 250-260.

Morrow, S. L., \& Smith, M. L. (1995). Constructions of survival and coping by women who have survived childhood sexual abuse. Journal of Counseling Psychology, 42, 24-33.

Ponterotto, J. G. (2005). Qualitative research in counseling psychology: A primer on research paradigms and philosophy of science. Journal of Counseling Psychology, 52, 126-136. 
Ponterotto, J. G., \& Grieger, I. (2008). Guidelines and competencies for cross-cultural counseling research. In P. B. Pedersen, J. G. Draguns, W. L. Lonner, \& J. E. Trimble (Eds.), Counseling across cultures ( $6^{\text {th }}$ ed., pp. 57-72). Thousand Oaks, CA: Sage.

Ponterotto, J. G., Mathew, J., \& Raughley, B. (2013). The value of mixed methods designs to social justice research in counseling and psychology. Journal for Social Action in Counseling and Psychology, 5(2), 42-68.

Sue, S. (1999). Science, ethnicity, and bias: Where have we gone wrong? American Psychologist, 54, 1070-1077.

Toporek, R. L., Gerstein, L. G., Fouad, N. A., Roysircar, G., \& Israel, T. (Eds.). (2006). Handbook of social justice in counseling psychology: Leadership, vision, and action. Thousand Oaks, CA: Sage.

Unger, R. K. (1983). Through the looking glass: No wonderland yet! (The reciprocal relationship between methodology and models of reality). Psychology of Women Quarterly, 8, 9-32. 